\title{
The Streptococcus anginosus species comprises five 165 rRNA ribogroups with different phenotypic characteristics and clinical relevance
}

\author{
Jan A. Jacobs, ${ }^{1}$ Corrie S. Schot ${ }^{2}$ and Leo M. Schouls ${ }^{2}$
}

Author for correspondence: Jan A. Jacobs. Tel: +31 4338746 44. Fax: +3143 3876643. e-mail: JJA@ms-azm-3.azm.nl

\footnotetext{
1 Department of Medical Microbiology, University Hospital of Maastricht, PO Box 5800, 6202 AZ Maastricht, The Netherlands

2 Department of Research Laboratory for Infectious Diseases, National Institute of Public Health and the Environment, PO Box 1, 3720 BA Bilthoven, The Netherlands
}

\begin{abstract}
A collection of 267 consecutively isolated Streptococcus anginosus strains was screened for the prevalence of previously described 'motile' strains by hybridization with oligonucleotide probes in a reverse line blot assay. The motile strains represented $101(37.8 \%)$ of the $S$. anginosus strains. The vast majority of these strains fermented mannitol and raffinose, whereas most other $S$. anginosus strains did not $(P<0.001)$. Most $(83 / 101)$ of the motile strains were recovered from the urogenital tract (including five strains from neonatal surveillance cultures) and only a minority (36) of them were associated with infection-related samples $(P<0.001)$. Strains that hybridized with the same oligonucleotide probes as the type strain S. anginosus ATCC $33397^{\top}$ ( = NCTC $10713^{\top}$ ) were designated ATCC-like strains. They accounted for $74(27.7 \%)$ of the strains examined, were commonly distributed among the different body parts and were significantly more associated with infectionrelated samples. Three other hybridization patterns were recognized in the reverse line blot assay, ribogroup I $(n=51)$, ribogroup II $(n=21)$ and ribogroup III $(n=19)$. Ribogroup II strains were significantly more frequently recovered from the abdominal cavity and were associated with infection-related samples. Ribogroup I included the majority of the $S$. anginosus strains that carried Lancefield group C. Comparison of the nearly complete 16S rRNA sequence of two representative strains of each ribogroup revealed that all five ribogroups were closely related ( $>97 \%$ sequence similarities), and that most sequence divergences between the ribogroups occurred in the 1024-1064 bp region of the 16S rRNA gene. The present data confirm the heterogeneity within the $S$. anginosus species.
\end{abstract}

Keywords: Streptococcus anginosus, 16S rRNA, motile strains

\section{INTRODUCTION}

In recent years, the taxonomy of the viridans streptococci has been clarified by the results of molecular typing techniques. Based on 16S rRNA sequence data, Kawamura et al. (1995) recognized six groups within the viridans streptococci, namely the 'pyogenic', 'mitis', 'salivarius', 'bovis', 'mutans' and 'anginosus' groups. The 'anginosus' group, which has previously

The GenBank accession numbers for the $16 \mathrm{~S}$ rRNA sequences of Streptococcus anginosus strains ATCC $33397^{\top}$, no. 1012, no. 367, no. 1204, no. 213 , no. 21 , no. 414, no. 1093, no. 1007 and no. 920 are AF104678, AF104679 and AF145239-AF145246, respectively. been referred to as the 'Streptococcus milleri' group, comprises a heterogeneous group of streptococcal species that may be implicated in purulent infections. Based on DNA-DNA reassociation studies and on phenotypic characteristics, Whiley \& Beighton (1991) gave an emended description of the three 'anginosus' group species as currently recognized: Streptococcus anginosus, Streptococcus constellatus and Streptococcus intermedius. The phylogenetic relatedness of these three species has been confirmed by $16 \mathrm{~S}$ rRNA sequence analysis (Bentley et al., 1991).

Recently, Bergman et al. (1995) described a distinct $16 \mathrm{~S}$ rRNA population within the 'anginosus' group. 
This rRNA population appeared to be most closely related to the $S$. anginosus species. Phenotypically, the strains belonging to this rRNA population displayed a gliding type of motility on certain types of chocolate agar, which is the reason why they were named 'motile' strains. The micromorphological patterns of the spreading zones were subsequently examined by Henrichsen (1997), who differentiated this type of socalled motility as a 'sliding type of spreading', in which the surface translocation is caused by reduced friction between the cells and substrate. Bergman et al. (1995) further noted that the spreading of the strains was not due to cellular organelles but appeared to be related to a high production of extracellular glycocalyx.

Genotypically, the motile strains showed a characteristic sequence signature in the 1050-1100 bp region of the 16S rRNA. From the viewpoint of pathogenicity, Bergman et al. (1995) suggested a potential advantage of the motile strains for colonization in vivo, as the production of extracellular glycocalyx might aid in adherence to mucosal surfaces. The limited number of motile strains included in their study, however, did not enable the relative importance of these strains in clinical samples to be estimated or possible associations with certain body sites or infections to be traced. Therefore, the present study was started in order to define the proportion of the motile strains within a collection of $S$. anginosus strains and to describe their clinical distribution and significance.

\section{METHODS}

Bacterial strains, species identification, phenotypic characterization and assessment of clinical significance. The streptococci studied were unique strains that were consecutively recovered from clinical specimens submitted for culture at the microbiological laboratory of the University Hospital of Maastricht. They were identified to the 'anginosus' group level if they produced acetoin in the Voges-Proskauer test, hydrolysed arginine and failed to utilize sorbitol. The strains were stored at $-70{ }^{\circ} \mathrm{C}$ on porous beads in cryopreservative (Microbank; Pro-Lab Diagnostics). Before inclusion in the study, strains were retrieved, checked for purity and subcultured to allow recovery from freezer storage. Haemolysis was assessed by observation of the subsurface growth in stabbed sheep blood agar plates. Both $\alpha$ - and $\gamma$-haemolysis were recorded as "non-haemolytic'. Lancefield grouping was performed on all isolates (Streptex; Murex Diagnostics). Sugar fermentation patterns were assessed with the API 20 Strep system (bioMérieux).

Strains were allocated to one of the following broad anatomical categories: head and neck region (including the oral cavity); thoracic cavity (including the respiratory tract); abdomen; genital tract; urinary tract; skin, bone and soft tissues; and the blood. Strains recovered from neonatal surveillance cultures were separately recorded but grouped together with the strains from the genital tract. Strains were considered infection-related based on clinical data (the information retrieved on the laboratory request form and in the hospital information system) and laboratory data (the kind of sample submitted and the predominance of leukocytes on the Gram stain). If clinical evidence was not conclusive or if the specimen may have been contaminated by saprophytic flora, the culture result was labelled as being of questionable clinical significance.

Sequencing of the 16S rRNA gene and reverse line blot hybridization. For further characterization on the 16S rRNA level, strains were retrieved on blood agar base (Oxoid) supplemented with sheep blood $5 \%(\mathrm{v} / \mathrm{v})$, checked for purity and streaked on secondary plates. The cells were transferred to a microcentrifuge tube and spun twice, first in a $100 \mu \mathrm{l}$ volume and subsequently in a $900 \mu \mathrm{l}$ volume of $10 \mathrm{mM}$ Tris, $150 \mathrm{mM} \mathrm{NaCl}$ buffer $\mathrm{pH} 8 \cdot 0$. The supernatant was discarded and the cells were resuspended in $100 \mu \mathrm{l}$ TE buffer (10 mM Tris/HCl, $1 \mathrm{mM}$ EDTA, pH 8.0) and heated at $99{ }^{\circ} \mathrm{C}$ for $10 \mathrm{~min}$.

Primers corresponding to residues 8-27 (primer 16S8FE) and 1523-1542 (biotin-labelled primer B-16S1523 RB) of the Escherichia coli 16S rRNA gene sequence were used to amplify the virtually complete $16 \mathrm{~S}$ rRNA gene sequence. Amplification was done in $25 \mu \mathrm{l}$ reaction volumes in an OmniGene Thermal Cycler (Hybaid). Each sample contained 10 pmol both primers, $0 \cdot 25$ units super Taq DNA polymerase (HT Biotechnology) and standard amounts of amplification reagents $(200 \mu \mathrm{M}$ of each deoxynucleoside triphosphate, $50 \mathrm{mM}$ Tris $/ \mathrm{HCl}, \mathrm{pH} 9.0,50 \mathrm{mM} \mathrm{KCl}$, $1.5 \mathrm{mM} \mathrm{MgCl}_{2}, 0 \cdot 01 \%$ gelatin, $0 \cdot 1 \%$ Triton X-100). A $25 \mu 1$ overlay of sterile mineral oil was added to the tubes. The

Table 1. Oligonucleotide sequences of primers and probes used in PCR and reverse line blot assay

\begin{tabular}{|c|c|c|c|}
\hline Designation & Target specificity & Nucleotide sequence & $\begin{array}{l}\text { Position in 16S } \\
\text { rRNA gene }\end{array}$ \\
\hline \multicolumn{4}{|l|}{ Primers for PCR: } \\
\hline $16 \mathrm{~S} 8 \mathrm{FE}$ & Eubacterial 16S rRNA gene & GGA ATT CAG AGT TTG ATC MTG GYT CAG & $8-27$ \\
\hline B-16S1523 RB & Eubacterial 16S rRNA gene & $\begin{array}{l}\text { 5'-Biotin-CGG GAT CCA AGG AGG TGA TCC ADC } \\
\text { CVC A }\end{array}$ & $1523-1542$ \\
\hline \multicolumn{4}{|c|}{ Amino-linked oligonucleotide probes for reverse line blotting: } \\
\hline A-Ang210 & S. anginosus ATCC $33397^{\mathrm{T}}$ & 5'-Amino-link-TGC AAT TGC ATC GCT AGT AG & $229-248$ \\
\hline A-Ang280 & S. anginosus ATCC $33397^{\mathrm{T}}$ & 5'-Amino-link-GTA ATG GCC TAC CTA GG & $281-297$ \\
\hline A-Ang 1000 & S. anginosus ATCC $33397^{\mathrm{T}}$ & 5'-Amino-link-TCC CGA TGC TAT TTC TAG A & $1019-1037$ \\
\hline A-MOT1000 & S. anginosus $\mathrm{X} 81023^{*}$ & 5'-Amino-link-CCC AGT GAC TGC CCT & $1020-1034$ \\
\hline
\end{tabular}

* According to Bergman et al. (1995). 
PCR programme used was $3 \mathrm{~min}$ at $94{ }^{\circ} \mathrm{C}$, followed by 25 cycles of amplification that consisted of $20 \mathrm{~s}$ at $94{ }^{\circ} \mathrm{C}, 1 \mathrm{~min}$ at $55^{\circ} \mathrm{C}$ and $1 \mathrm{~min}$ at $72^{\circ} \mathrm{C}$, and then by a final cycle of 7 min at $72^{\circ} \mathrm{C}$.

The PCR-amplified 16S rRNA genes were hybridized with 5 -amino-linked oligonucleotide probes (Isogen Bioscience). These probes were directed to species-specific regions of the 16S rRNA sequences of the type strains $S$. anginosus ATCC $33397^{\mathrm{T}}\left(=\right.$ NCTC $\left.10713^{\mathrm{T}}\right)$, S. constellatus ATCC $27823^{\mathrm{T}}(=$ NCTC $\left.11325^{\mathrm{T}}=\mathrm{NCDO} 2226^{\mathrm{T}}\right)$ and $S$. intermedius ATCC $27335^{\mathrm{T}}\left(=\right.$ NCTC $\left.11324^{\mathrm{T}}=\mathrm{NCDO} 2227^{\mathrm{T}}\right)$. The probes homologous to the sequences of the $S$. constellatus and the $S$. intermedius type strains are described elsewhere (Jacobs et al., 2000). The probes directed to the $S$. anginosus ATCC $33397^{\mathrm{T}} 16 \mathrm{~S}$ rRNA gene are listed in Table 1 . They were designated A-Ang210, A-Ang280 and A-Ang1000. In addition, a probe named A-MOT1000 was constructed to the subspecies-specific position on the 16S rRNA gene of the 'motile' strain X81023 described by Bergman et al. (1995). Hybridization reactions were performed in a reverse line blot assay as described by Kaufhold et al. (1994). In brief, oligonucleotide probes were covalently linked to an activated Biodyne $\mathrm{C}$ membrane (Pall Filtron) by the 5'-amino group link. This process was carried out in a miniblotter system (Immunetics). For hybridization, the membrane was again mounted into the miniblotter, in a $90^{\circ}$ rotated position, resulting in a perpendicular position of the slots on the lines which contained the oligonucleotide probes. The slots were filled with heat-denatured biotinylated PCR products and incubated at $42{ }^{\circ} \mathrm{C}$ for $60 \mathrm{~min}$ to allow hybridization. After hybridization, the slots were aspirated and the membranes were removed from the miniblotter and washed twice at $52{ }^{\circ} \mathrm{C}$ for $10 \mathrm{~min}$ with $2 \times \operatorname{SSPE}(360 \mathrm{mM}$ $\mathrm{NaCl}, 20 \mathrm{mM} \mathrm{Na}{ }_{2} \mathrm{HPO}_{4} \cdot \mathrm{H}_{2} \mathrm{O}, 2 \mathrm{mM}$ EDTA) $/ 0 \cdot 5 \%$ SDS.

Thereafter, the membranes were incubated with 1:4000 diluted streptavidin-peroxidase conjugate (Boehringer Mannheim) at $42{ }^{\circ} \mathrm{C}$ for $30 \mathrm{~min}$ in $10 \mathrm{ml} 2 \times \mathrm{SSPE} / 0.5 \%$ SDS and then washed twice (10 min each) with $150 \mathrm{ml} 2 \times$ SSPE- $0.5 \%$ SDS at $42{ }^{\circ} \mathrm{C}$. After a final 5 min wash step with $2 \times$ SSPE, the membranes were incubated with ECL detection reagents (Amersham) for $1 \mathrm{~min}$ and used to expose an ECL hyperfilm (Amersham) for $10 \mathrm{~min}$ to visualize hybridization. After each hybridization reaction, the PCR products were stripped from the membrane by washing it twice in $1 \% \mathrm{SDS}$ at $80{ }^{\circ} \mathrm{C}$ for $30 \mathrm{~min}$. and thereafter the membranes were incubated in $20 \mathrm{mM}$ EDTA pH $8 \cdot 0$, sealed moist in plastic and stored at $4{ }^{\circ} \mathrm{C}$ until reuse.

For DNA sequencing reactions, the fluorescence-labelled dideoxynucleotide technology was used (Perkin-Elmer). PCR products were purified using Qiaquick PCR purification kits (Qiagen). Sequence reactions were analysed on an ABI 377 automated DNA sequencer (Perkin-Elmer). Sequencing was performed using various $16 \mathrm{~S}$ rRNA specific primers. The collected sequences were assembled, edited and analysed with the DNASTAR package.

Statistical analysis. For statistical evaluation, proportions were compared by means of the chi-square test.

\section{RESULTS}

\section{Strains and characteristics}

Based on the results of the hybridizations in the reverse line blot assays, a total of $267 \mathrm{~S}$. anginosus strains (including $S$. anginosus strain ATCC $33397^{\mathrm{T}}$ ) were

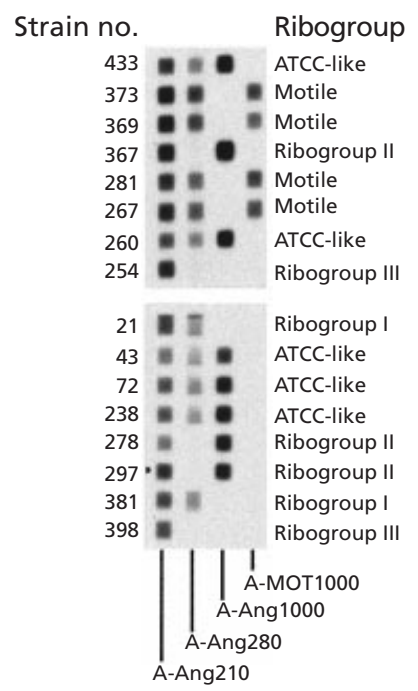

Fig. 1. Hybridization patterns of the different ribogroups within the $S$. anginosus species in the reverse line blot assay. Biotin-labelled PCR products of the nearly complete 16S rRNA gene of the strains (horizontal lanes) were hybridized with immobilized oligonucleotide probes (vertical lanes).

included in the study. From 261 strains, complete information on clinical significance and anatomical distribution was available. Most strains were recovered from the urogenital tract (109/261 strains, 41.7\%) and from the abdominal cavity $(85 / 261,32 \cdot 6 \%)$. The strains were recovered from 240 patients with a mean age of $49.7 \pm 21.6$ years and a male-to-female ratio of $1: 1 \cdot 09$.

\section{Patterns of hybridization}

Hybridization patterns are shown in Fig. 1. In the reverse line blot assay, 74 strains $(27.7 \%)$ reacted with the A-Ang210, A-Ang280 and A-Ang1000 probes but not with the A-MOT1000 probe. These strains encompassed the $S$. anginosus ATCC $33397^{\mathrm{T}}$ strain and were named 'ATCC-like strains'.

Another $101(37 \cdot 8 \%)$ strains reacted with the AAng210, A-Ang280 and A-MOT1000 probes but did not hybridize with the A-Ang 1000 probe: these strains were designated 'motile strains'. Apart from the ATCC-like strains and the motile strains, three other ribogroups with distinct reverse line blot hybridization patterns were observed. These groups were named ribogroup I, ribogroup II and ribogroup III, with representative strain numbers 213, 367 and 414, respectively. All three groups hybridized with the AAng210 probe, but none of them reacted with the AMOT 1000 probe. Ribogroup I and ribogroup II strains lacked reactivity with the A-Ang1000 probe and the AAng280 probe, respectively, and the ribogroup III strains lacked reactivity with both the A-Ang280 and A-Ang1000 probes. Representative strains of the five hybridization groups were run in each assay and they 
Table 2. Different ribogroups and their hybridization patterns observed in the reverse line blot assay for 267 S. anginosus strains

\begin{tabular}{|lccccc|}
\hline Probe reactivity & \multicolumn{5}{c|}{ Designation } \\
\cline { 2 - 6 } & $\begin{array}{c}\text { ATCC-like } \\
\text { strains }\end{array}$ & $\begin{array}{c}\text { Motile } \\
\text { strains }\end{array}$ & Ribogroup I & Ribogroup II & Ribogroup III \\
\hline A-Ang210 & + & + & + & + & + \\
A-Ang280 & + & + & + & - & - \\
A-Ang1000 & + & - & - & + & - \\
A-MOT1000 & - & + & - & - & - \\
Number of strains (\%) & $\mathbf{7 4 ( 2 7 \cdot 7 )}$ & $\mathbf{1 0 1 ~ ( 3 7 \cdot 8 )}$ & $\mathbf{2 2 ~ ( 8 \cdot 3 )}$ & $\mathbf{5 1 ( 1 9 \cdot 1 )}$ & $\mathbf{1 9 ( 7 \cdot 1 )}$ \\
\hline
\end{tabular}

Table 3. Lancefield groups and haemolytic pattern of the different ribosomal groups of $S$. anginosus strains

\begin{tabular}{|c|c|c|c|c|c|c|c|c|c|c|c|c|c|}
\hline \multirow{3}{*}{$\begin{array}{l}\text { Lancefield group } \\
\text { Haemolysis ... }\end{array}$} & \multicolumn{13}{|c|}{ Hybridization group } \\
\hline & \multicolumn{2}{|c|}{$\begin{array}{l}\text { ATCC-like } \\
\text { strains }\end{array}$} & \multicolumn{2}{|c|}{ Motile strains } & \multicolumn{2}{|c|}{ Ribogroup I } & \multicolumn{2}{|c|}{ Ribogroup II } & \multicolumn{2}{|c|}{ Ribogroup III } & \multicolumn{2}{|c|}{ Subtotal } & \multirow[t]{2}{*}{ Total (\%) } \\
\hline & - & + & - & + & - & + & - & + & - & + & - & + & \\
\hline Group A & 2 & 0 & 0 & 0 & 3 & 0 & 0 & 0 & 0 & 0 & 5 & 0 & $5(1 \cdot 9)$ \\
\hline Group C & 7 & 0 & 1 & 1 & 3 & 0 & 17 & 6 & 1 & 0 & 29 & 7 & $36(13 \cdot 5)$ \\
\hline Group F & 42 & 1 & 47 & 2 & 4 & 0 & 6 & 3 & 10 & 0 & 109 & 6 & $115(43 \cdot 1)$ \\
\hline Group G & 1 & 6 & 1 & 0 & 0 & 0 & 1 & 3 & 4 & 1 & 7 & 10 & $17(6 \cdot 4)$ \\
\hline No group & 14 & 1 & 49 & 0 & 11 & 1 & 8 & 7 & 3 & 0 & 85 & 9 & $94(35 \cdot 2)$ \\
\hline Total & 66 & 8 & 98 & 3 & 21 & 1 & 32 & 19 & 18 & 1 & 235 & 32 & 267 \\
\hline
\end{tabular}

displayed unequivocal hybridization patterns. Table 2 presents the five ribogroups, their hybridization pattern and the number of strains they included.

\section{Analysis of the 16S rRNA gene sequences of the various ribogroups}

The virtually complete $16 \mathrm{~S}$ rRNA sequences of two representative strains of each ribogroup were determined and compared to the 16S rRNA sequences of the $S$. constellatus ATCC $27823^{\mathrm{T}}$ and $S$. intermedius ATCC $27335^{\mathrm{T}}$ strains, which were previously deposited in GenBank under the accession numbers AF104676 and AF104671, respectively. Both strains of each ribogroup shared $99 \%$ sequence similarity and the calculated sequence divergences between the paired strains were due to the ambiguous sequence results or single base substitutions scattered along the entire 16S rRNA gene (approx. $1500 \mathrm{bp}$ ). Most sequence divergences between the distinct ribogroups occurred in the 1024-1064 bp region of the $S$. anginosus ATCC $33397^{\mathrm{T}}$ strain 16S rRNA gene. Within this particular region, three sequence patterns were recognized. One pattern was shared by the ATCC-like strains and the strains of ribogroup II, a second pattern was shared by the motile strains and the strains of ribogroup III, and a third pattern was displayed by the ribogroup I strains. Due to the substitution of a single base pair at position 285 of the $S$. anginosus ATCC $33397^{\mathrm{T}} 16 \mathrm{~S}$
rRNA gene, both ribogroup II and ribogroup III strains did not react with the A-Ang280 probe, whereas the other three ribogroups did hybridize with this probe. Within the $220-250 \mathrm{bp}$ region of the $S$. anginosus ATCC $33397^{\mathrm{T}}$ strain $16 \mathrm{~S}$ rRNA gene, no sequence divergences were observed between the distinct ribogroups.

\section{Phenotypic characteristics and anatomical distribution of the different ribogroups}

Table 3 lists the different ribogroups within the $S$. anginosus strains matched with the Lancefield groups and the haemolytic pattern. The majority $(98 / 101$, $97 \% ; P<0.001)$ of the motile strains were nonhaemolytic. The motile strains mainly encompassed strains carrying Lancefield group $\mathrm{F}$ or strains that were non-groupable $(P<0 \cdot 001)$. In contrast, 19 out of 51 $(37 \cdot 3 \%)$ ribogroup II strains were $\beta$-haemolytic and these 19 strains accounted for $59 \cdot 4 \%$ of all $32 \beta$ haemolytic $S$. anginosus strains $(P<0 \cdot 001)$. The ribogroup II strains further included the majority of the Lancefield group C $S$. anginosus strains (23/36 strains, $63.9 \% ; P<0.001)$; six of these 23 strains were $\beta$ haemolytic. Analysis of the sugar fermentation patterns revealed that the majority of the 101 motile strains fermented mannitol and raffinose $(87 \cdot 1 \%$ and $88.1 \%$, respectively), compared to only six strains out of the other ribogroups $(P<0 \cdot 001)$. 
Table 4. Anatomical origin and clinical significance of the different ribosomal groups of 263 S. anginosus strains

\begin{tabular}{|c|c|c|c|c|c|c|c|c|c|c|c|c|}
\hline \multirow{3}{*}{$\begin{array}{l}\text { Anatomical site } \\
\text { Clinical significance ...* }\end{array}$} & \multicolumn{12}{|c|}{ Hybridization group } \\
\hline & \multicolumn{2}{|c|}{$\begin{array}{c}\text { ATCC-like } \\
\text { strains }\end{array}$} & \multicolumn{2}{|c|}{ Motile strains } & \multicolumn{2}{|c|}{ Ribogroup I } & \multicolumn{2}{|c|}{ Ribogroup II } & \multicolumn{2}{|c|}{ Ribogroup III } & \multicolumn{2}{|c|}{ Subtotal } \\
\hline & $\mathbf{I}$ & $\mathbf{Q}$ & I & $\mathbf{Q}$ & I & $\mathbf{Q}$ & $\mathbf{I}$ & $\mathbf{Q}$ & $\mathbf{I}$ & $\mathbf{Q}$ & I & $\mathbf{Q}$ \\
\hline Abdominal cavity & 32 & 1 & 10 & 0 & 15 & 1 & 18 & 4 & 5 & 0 & 80 & 6 \\
\hline Skin, bone and soft tissues & 4 & 1 & 5 & 0 & 1 & 0 & 1 & 0 & 4 & 0 & 15 & 1 \\
\hline Thoracic cavity & 4 & 2 & 0 & 1 & 0 & 0 & 2 & 8 & 0 & 0 & 6 & 11 \\
\hline Head and neck & 3 & 3 & 1 & 0 & 0 & 0 & 1 & 3 & 2 & 1 & 7 & 7 \\
\hline Blood & 9 & 0 & 1 & 0 & 3 & 0 & 6 & 0 & 2 & 0 & 21 & 0 \\
\hline Genital tract & 0 & 3 & 7 & $29 \dagger$ & 0 & 0 & 0 & 5 & 1 & 0 & 8 & 37 \\
\hline Urinary tract & 3 & 6 & 12 & 35 & 1 & 0 & 1 & 2 & 2 & 2 & 19 & 45 \\
\hline Subtotal & 55 & 16 & 36 & 65 & 20 & 1 & 29 & 22 & 16 & 3 & 156 & 107 \\
\hline Total & \multicolumn{2}{|c|}{71} & \multicolumn{2}{|c|}{101} & \multicolumn{2}{|c|}{21} & \multicolumn{2}{|c|}{51} & \multicolumn{2}{|c|}{19} & \multicolumn{2}{|c|}{$263 t$} \\
\hline
\end{tabular}

* I, Infection-related; Q, questionable clinical significance.

$\dagger$ Including five strains recovered from neonatal surveillance cultures.

\$ The ATCC type strain $S$. anginosus $33397^{\mathrm{T}}$ was omitted and complete data were not available from three strains.

Table 4 lists the anatomical origin and the clinical significance of the strains belonging to the different ribogroups. The vast majority of the motile strains $(83 / 101,82 \cdot 2 \%)$ was recovered either from the urinary tract or from the genital tract $(P<0 \cdot 001)$, thereby accounting for $76 \cdot 1 \%$ of 109 S. anginosus strains that were recovered from these sites $(P<0 \cdot 001)$. The five $S$. anginosus strains recovered from neonatal surveillance cultures were identified as motile strains. Most (16/21, $82.2 \%$ ) strains belonging to ribogroup I were recovered from the abdominal cavity $(P<0 \cdot 001)$. The ATCC-like strains and the ribogroup II strains were more or less evenly distributed among the different body sites. The motile strains were significantly more frequently categorized as of questionable clinical significance, whereas the ATCC-like strains and nearly all ribogroup I strains were associated with infectionrelated samples $(P<0 \cdot 001)$. No relationship between pattern of haemolysis and clinical significance was found.

\section{DISCUSSION}

The $S$. anginosus species as currently defined by Whiley $\&$ Beighton (1991) is the most prevalent among the three 'anginosus' group (or ' $S$. milleri') species, accounting for $58 \cdot 7-64.4 \%$ of all 'anginosus' group strains recovered from consecutive clinical samples in the general hospital population (Gómez-Garcés et al., 1994; Jacobs et al., 1995; Bantar et al., 1996).

S. anginosus further represents the most common 'anginosus' group species among strains recovered from gastrointestinal and urogenital sources, and has been identified from most other anatomical sites (Whiley et al., 1992; Jacobs et al., 1995; Bantar et al., 1996). Most $S$. anginosus strains are non-haemolytic and they are less frequently associated with purulent infections as compared to the $S$. intermedius species (Jacobs et al., 1995).

Non-haemolytic strains that are able to acidify a wide sugar panel including mannitol and raffinose have been recognized within the 'anginosus' group for twenty years (Ball \& Parker, 1979). These so-called 'broadly fermentative strains' have been associated with the female genital tract and the urinary tract, and are generally considered as harmless contaminants (Ruoff \& Kunz, 1982; Ruoff et al., 1983). However, they may be implicated in neonatal infections associated with premature rupture of membranes and consequent ascending infection (Peck et al., 1987; Cox et al., 1987). The motile strains described by Bergman et al. (1995) phenotypically presented as broadly fermentative strains in their collection. This relation, however, was not exclusive, as the broadly fermentative $S$. anginosus strains also encompassed non-motile strains. Apart from the broadly fermentative strains, other phenotypic and genotypic subgroups have been detected within the S. anginosus species. They have been profoundly studied by Whiley et al. (1997). Within a reference collection of $21 \mathrm{~S}$. anginosus strains, these authors identified a previously undescribed species (i.e. $\beta$-haemolytic Lancefield group $\mathrm{C}$ strains, hyaluronidase-negative, $\beta$-galactosidase-positive) and recognized a distinct subspecies (i.e. $\beta$-haemolytic Lancefield group $\mathrm{C}$ strains, hyaluronidase-positive). Based on the results of DNA-DNA base pairing studies, the other $S$. anginosus strains studied by Whiley et al. (1997) formed a closely related group which included the strains that fermented mannitol and raffinose as well as the motile strains.

In the present study, the use of reverse line blotting enabled a large collection of consecutively isolated $S$. anginosus strains to be screened. The high number of 
clinical strains included made it possible to compare the relative prevalence of the distinct groups in the clinical setting. A drawback of this study was the lack of data on the ecology of the distinct groups in normal individuals or in healthy tissues. Moreover, only seven $\beta$-haemolytic Lancefield group $\mathrm{C}$ strains were included in this study, which represented a limited sample size as compared to the strain collections in reference laboratories.

Despite these limitations, it is clear that the motile strains constituted the most numerous population within the consecutive $S$. anginosus strains examined in our study. With an occasional exception, the motile strains were non-haemolytic. They either carried Lancefield group F or were non-groupable. The vast majority of them fermented mannitol and raffinose and, unlike the results of Bergman et al. (1995), this sugar fermentation pattern was almost completely confined to the motile strains. The motile strains represented the most common group of 'anginosus' group strains recovered from the urinary and genital tracts and accounted for all five 'anginosus' group strains recovered from neonatal surveillance cultures. Their presence in these sites might be explained by the production of extracellular glycocalyx that has been suggested to aid in the adherence to mucosal surfaces (Bergman et al., 1995). Only a limited fraction of motile strains was categorized as infection-related and this proportion was even lower if only the urogenital sites were considered. This apparent low pathogenicity could be ascribed to the lack of invasive properties displayed by the motile strains. However, it might also be explained by the fact that the urogenital tract is less prone to local mucosal trauma as compared to other sites, e.g. the gastrointestinal tract. Indeed, local trauma such as ulceration, perforation or surgery constitutes a predisposing factor for invasive and purulent infections by 'anginosus' group strains (Gossling, 1988; Jacobs et al., 1994).

The motile strains recovered from the gastrointestinal tract were all infection-related. The ten motile strains assigned to the gastrointestinal tract were recovered as part of a mixed flora, six of them from perirectal abscesses and one strain from a pelvic abscess.

Apart from the motile strains, four other ribogroups were demonstrated. The ATCC-like strains, which included the $S$. anginosus ATCC $33397^{\mathrm{T}}$ type strain, represented only $27 \cdot 7 \%$ of all S. anginosus strains. At the phenotypic level, no single phenotypic characteristic or any combination of haemolytic activity, Lancefield group or API profile was confined to any of the five ribogroups detected. Six out of seven $\beta$ haemolytic Lancefield group C S. anginosus strains belonged to the ribogroup II group and could be identical to the $\beta$-galactosidase-positive strains of Lancefield group $C$ that were recently described by Whiley et al. (1997). However, the $\beta$-galactosidase activity of these strains could not be compared with that in the data reported by Whiley et al. (1997) because the API 20 Strep gallery only accommodates the $\beta$-galactosidase naphtholic substrate and not the chromogenic oxidase substrate which is the only suitable one for the detection of $\beta$-galactosidase activity in 'anginosus' group strains (Whiley et al., 1997). The rather poor phenotypic consistency of the ATCC-like group and ribogroups I, II and III makes their existence as separate subspecies debatable. On the other hand, it is striking that the ribogroup I strains and the ATCC-like strains were significantly more associated with infection-related samples. As suggested previously, a study on the ecology of these strains in healthy persons should provide a more accurate denominator for comparison.

Alignment of the nearly complete $16 \mathrm{~S}$ rRNA sequences of the five ribogroups showed that each group displayed less than $97 \%$ sequence similarity with the $S$. constellatus ATCC $27823^{\mathrm{T}}$ and $S$. intermedius ATCC $27335^{\mathrm{T}}$ strains and this low degree of sequence similarity indicates different genomic species (Stackebrandt \& Goebel, 1994). In contrast, all five ribogroups shared $>97 \%$ sequence similarity with the S. anginosus ATCC $33397^{\mathrm{T}}$ strain. The motile strains displayed similarity levels of $97.8 \%$ and $98.1 \%$ with the S. anginosus ATCC $33397^{\mathrm{T}}$ strain but, as cited above, Whiley et al. (1997) found no genotypic or phenotypic arguments for considering these strains as a distinct subspecies. The fact that most sequence divergences occurred within bp 1024-1064 of the $S$. anginosus ATCC $33397^{\mathrm{T}}$ 16S rRNA gene explains the apparent homogeneity within the $S$. anginosus strains that has been reported previously (Jacobs et al., 1996). In the latter study, line blotting was performed with diagnostic oligonucleotide probes directed to the $b p$ 213-231 region of the 16S rRNA genes of the three 'anginosus' group ATCC type strains. Within this particular region, the three 'anginosus' group species carry different sequence signatures and the five $S$. anginosus ribogroups presently recognized display complete sequence identity.

In conclusion, the present study demonstrated five distinct ribogroups within the $S$. anginosus species. The motile strains were the most numerous group. These strains encompassed the vast majority of the broadly fermentative strains, showed a predilection for the urogenital tract and were recovered from samples of questionable clinical significance. The other ribogroups were more commonly distributed among the different body sites. The ATCC-like strains (which included the $S$. anginosus ATCC $33397^{\mathrm{T}}$ strain) and the ribogroup I strains were most frequently associated with infection-related samples. All five ribogroups were closely related at the $16 \mathrm{~S}$ rRNA level and displayed $>97 \%$ sequence similarity. The present findings confirm the heterogeneity within the $S$. anginosus species.

\section{REFERENCES}

Ball, L. C. \& Parker, M. T. (1979). The cultural and biochemical characters of Streptococcus milleri strains isolated from human sources. J Hyg 82, 63-78. 
Bantar, C., Fernandez Canigia, L., Rellosa, S., Lanza, A., Bianchini, H. \& Smayevski, J. (1996). Species belonging to the 'Streptococcus milleri' group: antimicrobial susceptibility and comparative prevalence in significant clinical specimens. J Clin Microbiol 34, 2020-2022.

Bentley, R. W., Leigh, J. A. \& Collins, M. D. (1991). Intrageneric structure of Streptococcus based on comparative analysis of small-subunit rRNA sequences. Int J Syst Bacteriol 41, 487-494.

Bergman, S., Selig, M., Collins, M. D., Farrow, J. A. E., Baron, E. J., Dickersin, G. R. \& Ruoff, K. L. (1995). 'Streptococcus milleri' strains displaying a gliding type of motility. Int $J$ Syst Bacteriol 45, 235-239.

Cox, R. A., Chen, K., Coykendall, A. L., Wesbecher, P. \& Herson, V. C. (1987). Fatal infection in neonates of 26 weeks' gestation due to Streptococcus milleri: report of two cases. J Clin Pathol 40, 190-193.

Gómez-Garcés, J.-L., Alós, J.-I. \& Cogollos, R. (1994). Bacteriologic characteristics and antimicrobial susceptibility of 70 clinically significant isolates of Streptococcus milleri group. Diagn Microbiol Infect Dis 19, 69-73.

Gossling, J. (1988). Occurrence and pathogenicity of the Streptococcus milleri group. Rev Infect Dis 10, 257-285.

Henrichsen, J. (1997). 'Streptococcus milleri' strains exhibit not gliding motility but sliding. Int J Syst Bacteriol 47, 604.

Jacobs, J. A., Pietersen, H. G., Stobberingh, E. E. \& Soeters, P. B. (1994). Bacteremia due to the 'Streptococcus milleri' group: analysis of 19 cases. Clin Infect Dis 19, 704-713.

Jacobs, J. A., Pietersen, H. G., Stobberingh, E. E. \& Soeters, P. B. (1995). Streptococcus anginosus, Streptococcus constellatus and Streptococcus intermedius: clinical relevance, haemolytic and serologic characteristics. Am J Clin Pathol 104, 547-553.

Jacobs, J. A., Schot, C. S., Bunschoten, A. E. \& Schouls, L. M. (1996). Rapid species identification of 'Streptococcus milleri' by line blot hybridization: identification of a distinct 16S rRNA population closely related to Streptococcus constellatus. J Clin Microbiol 34, 1717-1721.
Jacobs, J. A., Schot, C. S. \& Schouls, L. M. (2000). Haemolytic activity of the 'Streptococcus milleri group' and relationship between haemolysis restricted to human red blood cells and in Streptococcus intermedius. J Med Microbiol 49, 55-62.

Kaufhold, A., Potbielski, A., Baumgarten, G., Blokpoel, M., Top, J. \& Schouls, L. M. (1994). Rapid typing of group A streptococci by use of DNA amplification and nonradioactive allele specific oligonucleotide probes. FEMS Microbiol Lett 119, 19-26.

Kawamura, Y., Hou, X.-G., Sultana, F., Miura, H. \& Ezaki, T. (1995). Determination of 16S rRNA sequences of Streptococcus mitis and Streptococcus gordonii and phylogenetic relationships among members of the genus Streptococcus. Int J Syst Bacteriol 45, 406-408.

Peck, G., Coe, P. R. \& Allen, J. B. (1987). Fatal infection in neonates caused by $S$. milleri. J Clin Pathol 40, 1386.

Ruoff, K. L. \& Kunz, L. J. (1982). Identification of viridans streptococci isolated from clinical specimens. J Clin Microbiol 15, 920-925.

Ruoff, K. L., Fishman, J. A., Calderwood, S. B. \& Kunz, L. J. (1983). Distribution and incidence of viridans streptococcal species in routine clinical specimens. Am J Clin Pathol 80, 854-858.

Stackebrandt, E. \& Goebel, B. M. (1994). Taxonomic note: a place for DNA-DNA reassociation and 16S rRNA sequence analysis in the present species definition in bacteriology. Int $J$ Syst Bacteriol 44, 846-849.

Whiley, R. A. \& Beighton, D. (1991). Emended descriptions and recognition of Streptococcus constellatus, Streptococcus intermedius and Streptococcus anginosus as distinct species. Int $J$ Syst Bacteriol 41, 1-5.

Whiley, R. A., Beighton, D., Winstanley, T. G., Frazer, H. Y. \& Hardie, J. M. (1992). Streptococcus intermedius, Streptococcus constellatus, and Streptococcus anginosus (the Streptococcus milleri group): association with different body sites and clinical infections. J Clin Microbiol 30, 243-244.

Whiley, R. A., Hall, L. M. C., Hardie, J. M. \& Beighton, D. (1997). Genotypic and phenotypic diversity within Streptococcus anginosus. Int J Syst Bacteriol 47, 645-650. 